\title{
Aplasia Ras homologue member I overexpression inhibits tumor growth and induces apoptosis through inhibition of PI3K/Akt survival pathways in human osteosarcoma MG-63 cells in culture
}

\author{
KAISHAN YE, SHUANKE WANG, YONG YANG, XUEWEN KANG, JING WANG and HUA HAN \\ Department of Orthopaedics, Lanzhou University Second Hospital, Lanzhou, Gansu 730030, P.R. China
}

Received December 16, 2014; Accepted June 26, 2015

DOI: $10.3892 /$ ijmm.2015.2278

\begin{abstract}
Aplasia Ras homologue member I (ARHI), an imprinted tumor-suppressor gene, is downregulated in various types of cancer. However, the expression, function and specific mechanisms of ARHI in human osteosarcoma (OS) cells remain unclear. The aim of the present study was to assess the effect of ARHI on OS cell proliferation and apoptosis and its associated mechanism. In the study, ARHI mRNA and protein levels were markedly downregulated in OS cells compared with the human osteoblast precursor cell line hFOB1.19. By generating stable transfectants, ARHI was overexpressed in OS cells that had low levels of ARHI. Overexpression of ARHI inhibited cell viability and proliferation and induced apoptosis. However, caspase-3 activity was not changed by ARHI overexpression. In addition, phosphorylated Akt protein expression decreased in the ARHI overexpression group compared to that in the control vector group. The knockdown of ARHI also resulted in the promotion of cell proliferation and the attenuation of apoptosis in MG-63 cells. Additionally, ARHI silencing increased the level of p-Akt. The present results indicate that ARHI inhibits OS cell proliferation and may have a key role in the development of OS.
\end{abstract}

\section{Introduction}

Osteosarcoma (OS), the most common primary malignant solid bone tumor, is the second leading cause of cancer-related fatality in children and young adults (1-3). OS are high-grade

Correspondence to: Dr Kaishan Ye, Department of Orthopaedics, Lanzhou University Second Hospital, 82 Cuiyingmen, Chengguan, Lanzhou, Gansu 730030, P.R. China

E-mail: kaishanye@126.com

Abbreviations: ARHI, aplasia Ras homologue member I; OS, osteosarcoma; RT-qPCR, reverse transcription-quantitative polymerase chain reaction

Key words: osteosarcoma, aplasia Ras homologue member I, proliferation, apoptosis, PI3K/Akt aggressive tumors that comprise $\sim 20 \%$ of all primary bone cancers and $2.4 \%$ of all malignancies in pediatric patients $(4,5)$. Currently, although several conventional therapies are offered for the clinical treatment of OS, such as radiation, chemotherapy, surgical resection, or combinations of chemotherapy and radiation, therapeutic outcomes for OS are not satisfactory (6). Evidence suggests that the therapeutic options for OS have not improved over the past four decades $(7,8)$; thus, novel approaches to OS are required. Efforts to identify new genes and signaling pathways that affect tumor progress have suggested that the aplasia Ras homologue member I (ARHI) gene (also known as DIRAS3) has a crucial role in numerous types of cancer, such as breast cancer $(9,10)$, ovarian cancer $(11,12)$, hepatocellular carcinoma (13), lung cancer (14) and glioma (15). Additionally, it has been reported that the ARHI protein is downregulated in multiple myeloma endothelial cells and is involved in proliferation and signal transduction in these cells (16). However, the specific functions of ARHI in the progression of OS remain unclear.

ARHI, a newly discovered, maternally endogenous imprinted tumor-suppressor gene, is a $26-\mathrm{kDa}$ small GTP-binding protein-encoding gene that is located on human chromosome 1p31 (17). Although ARHI is a member of the Ras superfamily and shares $60 \%$ homology with Ras and Rap, it has been identified as a tumor-suppressor gene that suppresses tumor cell growth (18). ARHI has attracted increasing attention in tumor research since evidence was reported indicating that ARHI could inhibit tumor cell proliferation (19). Accumulating evidence has shown that ARHI has an important role in the occurrence, metastasis, invasion and development of cancers (20-22). In addition, numerous types of cancer have low or absent levels of ARHI $(23,24)$. Increased expression of ARHI in pancreatic cancer cells can inhibit cell proliferation by acting on cell cycle genes $(19,21,25)$. It is now well established that ARHI is most highly expressed in normal breast and ovarian tissues, whereas the ARHI level in breast and ovarian cancer is significantly reduced $(17,26)$. Another study also demonstrated that high levels of ARHI inhibit tumor growth and angiogenesis in hepatocellular carcinoma (27).

Although ARHI has a number of positive effects in numerous types of tumor, it remains to be established whether ARHI is expressed in OS cells, and whether ARHI overexpression is associated with cell growth and apoptosis. The 
present study shows for the first time that overexpression of the ARHI gene induced by cellular transfection significantly curbs the growth of OS cells and induces cell apoptosis. ARHI overexpression inhibits OS cell proliferation and induces cell apoptosis through the phosphatidylinositol3-kinase (PI3K)/Akt signaling pathway. Additionally, the study provides evidence that ARHI could serve as a target for OS and upregulation of ARHI may be a useful method in treating OS in humans.

\section{Materials and methods}

Antibodies. Mouse anti-ARHI monoclonal antibody (ab45768), mouse anti- $\beta$-actin monoclonal antibody (ab6276) and horseradish peroxidase (HRP)-conjugated rabbit anti-mouse immunoglobulin G (IgG; ab6728) were all obtained from Abcam (Cambridge, MA, USA).

Cell culture and transfection. Three human OS cell lines, MG-63, U2OS and SAOS-2, were used in the study. All the cell lines were obtained from the American Type Culture Collection (Rockville, MD, USA). All the OS cells were cultured in Dulbecco's modified Eagle's medium (DMEM; Mediatech, Inc., Herndon, VA, USA) supplemented with $10 \%$ fetal bovine serum (FBS; Gibco-BRL, Gaithersburg, $\mathrm{MD}, \mathrm{USA}$ ) in a humidified atmosphere at $37^{\circ} \mathrm{C}$ supplemented with $5 \% \quad \mathrm{CO}_{2}$. The human osteoblast precursor cell line hFOB1.19 was cultured in DMEM/F12 medium supplemented with $10 \% \mathrm{FBS}$ at $37^{\circ} \mathrm{C}$ supplemented with $5 \% \mathrm{CO}_{2}$. Cells in the exponential growth phase were used in the study. Cell transfection was performed with either human ARHI cDNA (pcDNA3.1-ARHI) or the control vector pcDNA3.1 using Lipofectamine 2000 (Invitrogen Life Technologies, Carlsbad, CA, USA), according to the manufacturer's instructions. The cells transfected with pcDNA3.1-ARHI were harvested and measured by reverse transcription-quantitative polymerase chain reaction (RT-qPCR) and western blot analysis.

Cell viability. MG-63, U2OS and SAOS-2 cell viability was assessed by the trypan blue exclusion assay, as previously described with minor modifications (28). Briefly, transfected cells and control cells were seeded at $2 \times 10^{4}$ cells/well on 96-well plates using DMEM and were incubated for different periods of time $(0,24,48,72$ and $96 \mathrm{~h})$. Cells were subsequently stained with trypan blue (40 $\mu \mathrm{l}$; Sigma, St. Louis, MO, USA) and counted using a light microscope.

3-(4,5-dimethylthiazol-2-yl)-2,5-diphenyltetrazolium bromide (MTT). MG-63 cells were transfected as described above and seeded at $2 \times 10^{4}$ cells/well on 24 -well plates. After culturing for $72 \mathrm{~h}$, the live cells were measured using a cell proliferation MTT kit (Chemicon International, Inc., Temecula, CA, USA) by measuring MTT cleavage of active mitochondria, according to the manufacturer's instructions.

Flow cytometric analysis of apoptosis. MG-63 cell apoptosis was analyzed using flow cytometry (Beckman Coulter, Inc., Brea, CA, USA). Briefly, $2 \times 10^{4}$ cells/well were seeded in 96-well plates. Cells were incubated with Annexin V and propidium iodide (PI) for $20 \mathrm{~min}$ at room temperature. Apoptotic MG-63 cells were subsequently detected by flow cytometry.
Total RNA extraction and RT-qPCR. The RNA extraction and RT-qPCR procedure were performed as previously described $(29,30)$. Briefly, total RNA was extracted from OS cells using the TRIzol ${ }^{\circledR}$ reagent (Invitrogen Life Technologies) according to the manufacturer's instructions. RT-qPCR was carried out using the SYBR-Green ReadyMix on an ABI PRISM 7000 Sequence Detection system (both from Applied Biosystems, Foster City, CA, USA). The primer sequences (13) used for RT-qPCR were as follows: ARHI forward, 5'-CAGCTGGTTTCTTACCACGTAT-3' and reverse, 5'-GCACAAGTTCTCCCACACTTAG-3'; and $\beta$-actin forward, 5'-TCACCCACACTGTGCCCATCTACGA-3' and reverse, 5'-CAGCGGAACCGCTCATTGCCAATGG-3'. The primers used were all synthesized by Sangon Biotech Co., Ltd. (Shanghai, China). Relative expression of the ARHI gene was calculated using the $2^{-\Delta \Delta C T}$ method (31). The level of $\beta$-actin mRNA was used as the internal control.

Western blot analysis. ARHI protein expression in OS cells was determined according to a previously described method with minor modifications (15). Total protein was isolated from each group of OS cells using radioimmunoprecipitation assay lysis buffer, and the protein concentration was determined using a bicinchoninic acid assay kit (Beyotime, Jiangsu, China). Protein samples $(20 \mu \mathrm{g} /$ lane $)$ were separated by $10 \%$ SDS-PAGE and electrophoretically transferred to nitrocellulose membranes. After blocking overnight at $4^{\circ} \mathrm{C}$ with $5 \%$ skimmed milk, the resulting blots were first probed with mouse anti-ARHI antibody $(1: 1,000)$, and subsequently with HRP-conjugated rabbit anti-mouse $\operatorname{IgG}(1: 4,000)$, followed by detection using the ECL reagent (Boehringer Mannheim GmbH, Mannheim, Germany).

Small interfering RNA (siRNA) transfection. OS MG-63 cells with the ARHI protein were transfected with ARHI siRNA (siARHI) or the control siRNA (siMock) using the DharmaFECT 4 Transfection reagent (GE Dharmacon, Lafayette, CO, USA), as previously described (20,32). Briefly, $100 \mathrm{nM}$ of the siRNA mixture with transfection reagents was co-incubated at room temperature for $20 \mathrm{~min}$. Subsequently, the mixture was added to MG-63 cells for $48 \mathrm{~h}$. The cells were harvested and the measurement of mRNA and protein expression was performed by RT-qPCR and western blot analysis, respectively. siARHI- and siMock-transfected cells were used for further studies.

Caspase-3 activity assay. The activity of caspase-3 in OS MG-63 cells was assayed using the Caspase-Glo ${ }^{\circledR}$ 3/7 assay (Promega Corp., Madison, WI, USA) according to the manufacturer's instructions (33). Briefly, MG-63 cells were seeded in 24-well plates and were subsequently treated with the Caspase-Glo ${ }^{\circledR} 3 / 7$ reagent $(50 \mathrm{ml})$ for $30 \mathrm{~min}$. Luminescence was measured on a TECAN GENios Pro microplate reader. Caspase activity was measured after $72 \mathrm{~h}$ for cells treated with Ad-ARHI and after $48 \mathrm{~h}$ for cells treated with siARHI.

Statistical analysis. The data were analyzed using SPSS 13.0 statistical analysis software (SPSS, Inc., Chicago, IL, USA). The differences between two groups were compared by Dunnett's test. $\mathrm{P}<0.05$ was considered to indicate a statistically significant difference. 
A

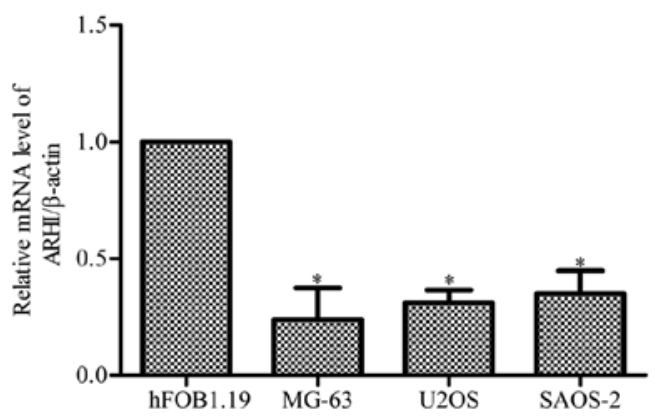

B

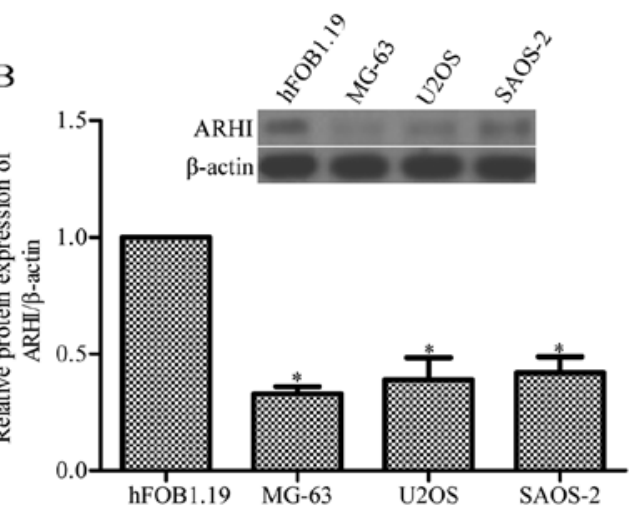

Figure 1. Aplasia Ras homologue member I (ARHI) mRNA and protein expression in osteosarcoma (OS) cell lines MG-63, U2OS, SAOS-2. (A) Reverse transcription-quantitative polymerase chain reaction detected the mRNA level of ARHI. (B) ARHI protein expression profiles in hFOB1.19 and human OS cell lines, including MG-63, U2OS and SAOS-2, were examined by western blot analysis. The experiments were repeated at least three times. Values obtained from three individual experiments are expressed as mean \pm standard deviation, ${ }^{*} \mathrm{P}<0.05$ vs. control.

A

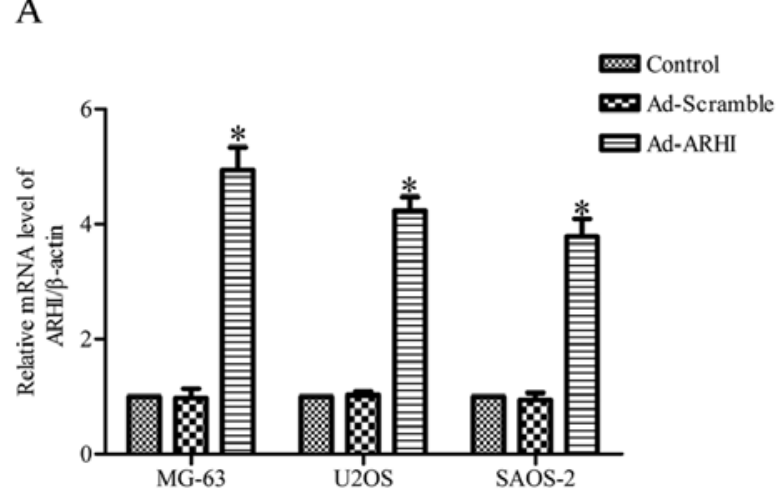

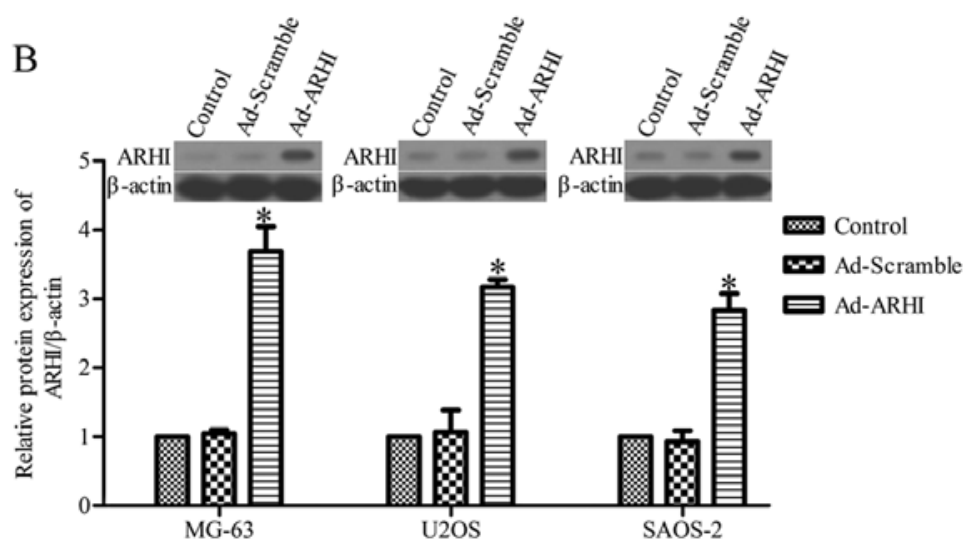

Figure 2. Overexpression of aplasia Ras homologue member I (ARHI) in human osteosarcoma (OS) cells. (A) ARHI mRNA and (B) protein expression in the OS cell lines, including MG-63, U2OS and SAOS-2, were measured by reverse transcription-quantitative polymerase chain reaction and western blot analysis. The experiments were repeated at least three times. Values obtained from three individual experiments are expressed as mean \pm standard deviation, "P<0.05 vs. control. Control, cells without treatment; Ad-Scramble, cells transfected with null vector; Ad-ARHI, cells transfected with ARHI overexpression vector.

\section{Results}

ARHI mRNA and protein expression is markedly decreased in OS cell lines. The evidence indicates that the expression of ARHI is reduced in numerous types of cancer and a high level of ARHI inhibits tumor growth (13); however, the level and the function of ARHI in OS remain unclear. Whether ARHI also has a role in OS cell growth was determined. As a result of the RT-qPCR and western blot analysis on the three OS cell lines, MG-63, U2OS and SAOS-2, the data show that the expression of ARHI was markedly decreased compared with the human osteoblast precursor hFOB1.19 ( $\mathrm{P}<0.05$; Fig. 1).

Overexpression of ARHI inhibits cell viability and proliferation. In order to assess the effects of ARHI on OS cell viability and cell growth, stable upregulation of ARHI in the OS cell lines, MG-63, U2OS and SAOS-2, using pcDNA3.1-ARHI was constructed. RT-qPCR and western blot analysis results showed that the level of ARHI mRNA and protein in the three OS cell lines transfected with Ad-ARHI were much higher compared with the control group $72 \mathrm{~h}$ later $(\mathrm{P}<0.05)$ (Fig. 2A and $\mathrm{B}$ ), whereas ARHI expression was not significantly different between the Ad-Mock group and the control group ( $\mathrm{P}>0.05)$ (Fig. 2A and B). The mRNA and protein expression were also upregulated in MG-63, U2OS and SAOS-2 cells transfected with pcDNA3.1-ARHI for 24, 48 and $96 \mathrm{~h}$ (data not shown).

Overexpression of ARHI inhibits cell viability and proliferation. To determine the effect of ARHI overexpression on the viability of OS cells, the trypan blue exclusion assay was performed on all the groups at various post-transfection time-points. The results show that Ad-AHRI-expressing MG-63, U2OS and SAOS-2 cell viability was significantly lower compared with the respective cells in the Ad-Mock and control groups $(\mathrm{P}<0.05$; Fig. 3A-C).

Subsequently, the effect of ARHI overexpression for $72 \mathrm{~h}$ on the growth of MG-63, U2OS and SAOS-2 cells was determined by the MTT method. The results indicate that the growth of Ad-AHRI-expressing MG-63, U2OS and SAOS-2 cells was markedly decreased compared to the respective cells in the control groups $(\mathrm{P}<0.05$; Fig. 3D-F). However, no significant effects were observed in cells treated with Ad-Mock (Fig. 3D-F). These results suggest that ARHI overexpression significantly inhibits the viability and growth of OS cells. 

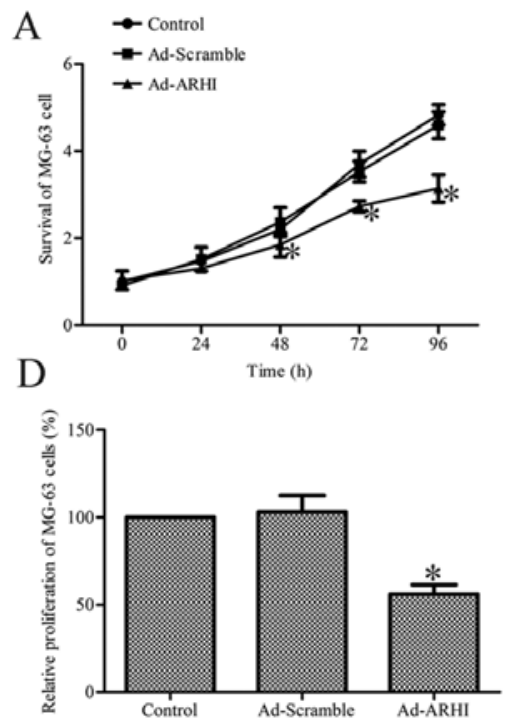
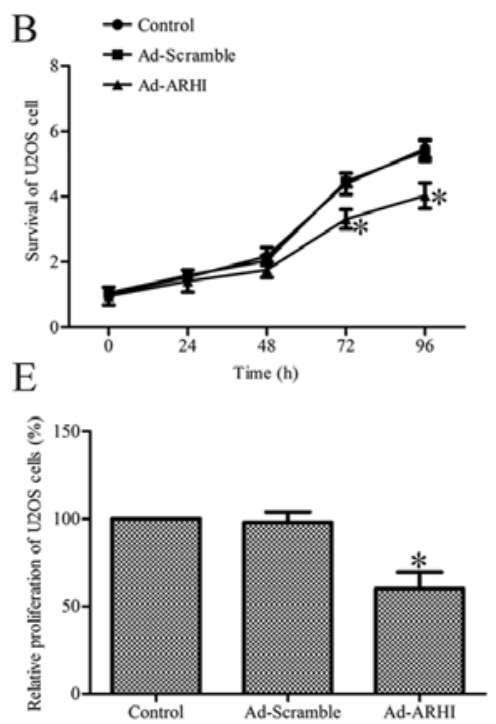
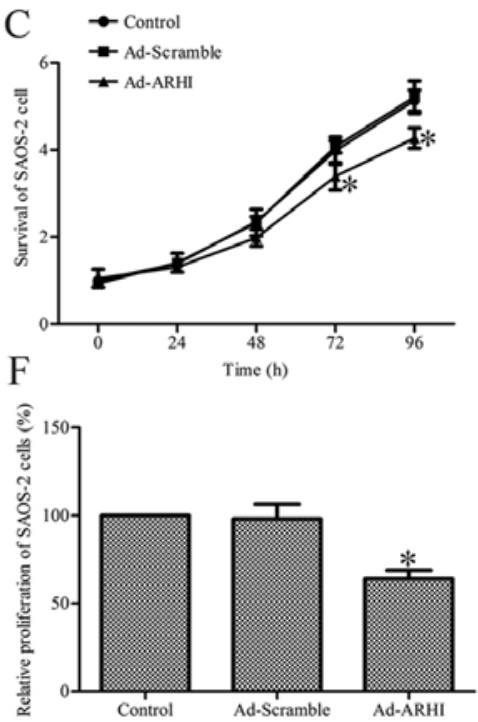

Figure 3. Effects of aplasia Ras homologue member I (ARHI) on cell viability and proliferation. Cell viability of (A) MG-63, (B) U2OS and (C) SAOS-2 cells was measured by trypan blue exclusion at various post-transfection time-points $(0,24,48,72$ and $96 \mathrm{~h}$ ). Proliferation of (D) MG-63, (E) U2OS and (F) SAOS-2 cells was analyzed by the 3-(4,5-dimethylthiazol-2-yl)-2,5-diphenyltetrazolium bromide assay $72 \mathrm{~h}$ after Ad-ARHI transfection. The experiments were repeated at least three times. Values obtained from three individual experiments are expressed as mean \pm standard deviation, ${ }^{*} \mathrm{P}<0.05$ vs. control.

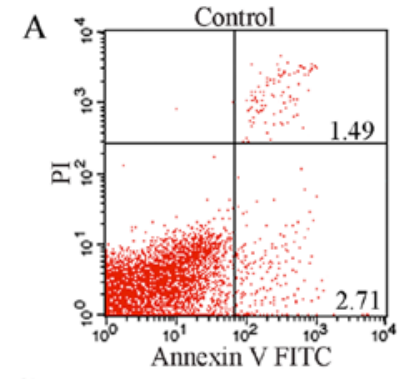

B

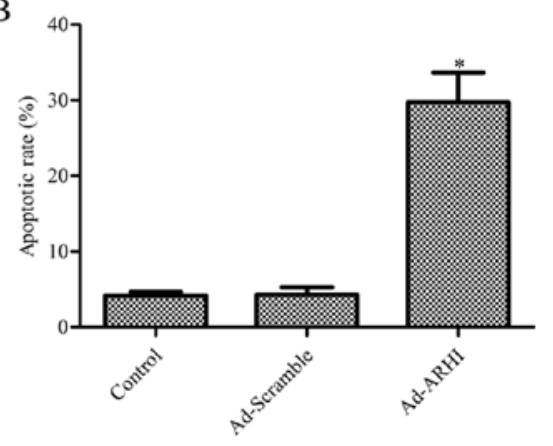

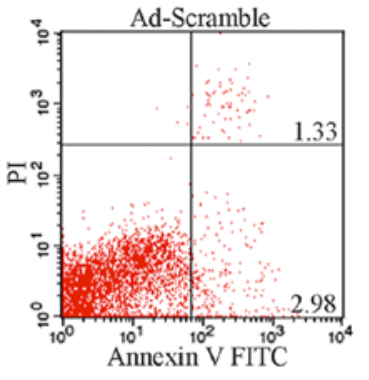

C
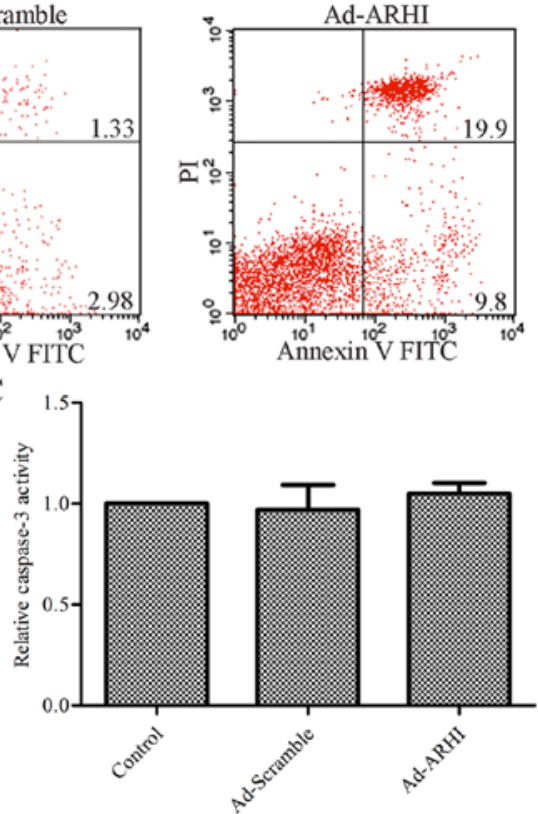

Figure 4. Effects of aplasia Ras homologue member I (ARHI) on MG-63 cell apoptosis and caspase-3 activity. (A) ARHI overexpression MG-63 cell apoptosis measured by flow cytometric analysis. Cells were stained with Annexin V and propidium iodide (PI). MG-63 cells were transfected with Ad-ARHI or the control Ad-Scramble for $72 \mathrm{~h}$. (B) Quantitative analyses of the MG-63 cell apoptotic rate. (C) Caspase-3 activation in MG-63 cells. Caspase-3 activation was detected by the Caspase-Glo ${ }^{\circledast} 3 / 7$ assay. The experiments were repeated at least three times. Values obtained from three individual experiments are expressed as mean \pm standard deviation, ${ }^{*} \mathrm{P}<0.05$ vs. control.

Effect of ARHI overexpression on MG-63 cell apoptosis. To further determine the mechanism by which ARHI contributes to reduced survival in OS cells, the effect of ARHI on MG-63 cell apoptosis was assessed by flow cytometric analysis. The results indicate that the rate of apoptosis was significantly higher with ARHI overexpression compared with the control group $(\mathrm{P}<0.05$; Fig. $4 \mathrm{~A}$ and $\mathrm{B})$, whereas there was no evident difference between the Ad-Mock group and the control group ( $\mathrm{P}>0.05$; Fig. 4A and B). A total of $4.2 \%$ apoptotic cells in the control group, and 4.3 and $29.7 \%$ apoptotic cells were observed in the Ad-ARHI and Ad-Mock group, respectively. Subsequently, the activity of caspase- 3 was determined in the Ad-ARHI, Ad-Mock and control groups by the Caspase-Glo ${ }^{\circledR}$ 3/7 assay. As shown in Fig. 4C, the activity of caspase- 3 had no significantly change in the Ad-ARHI group compared with the Ad-Mock and control groups $(\mathrm{P}>0.05)$. 

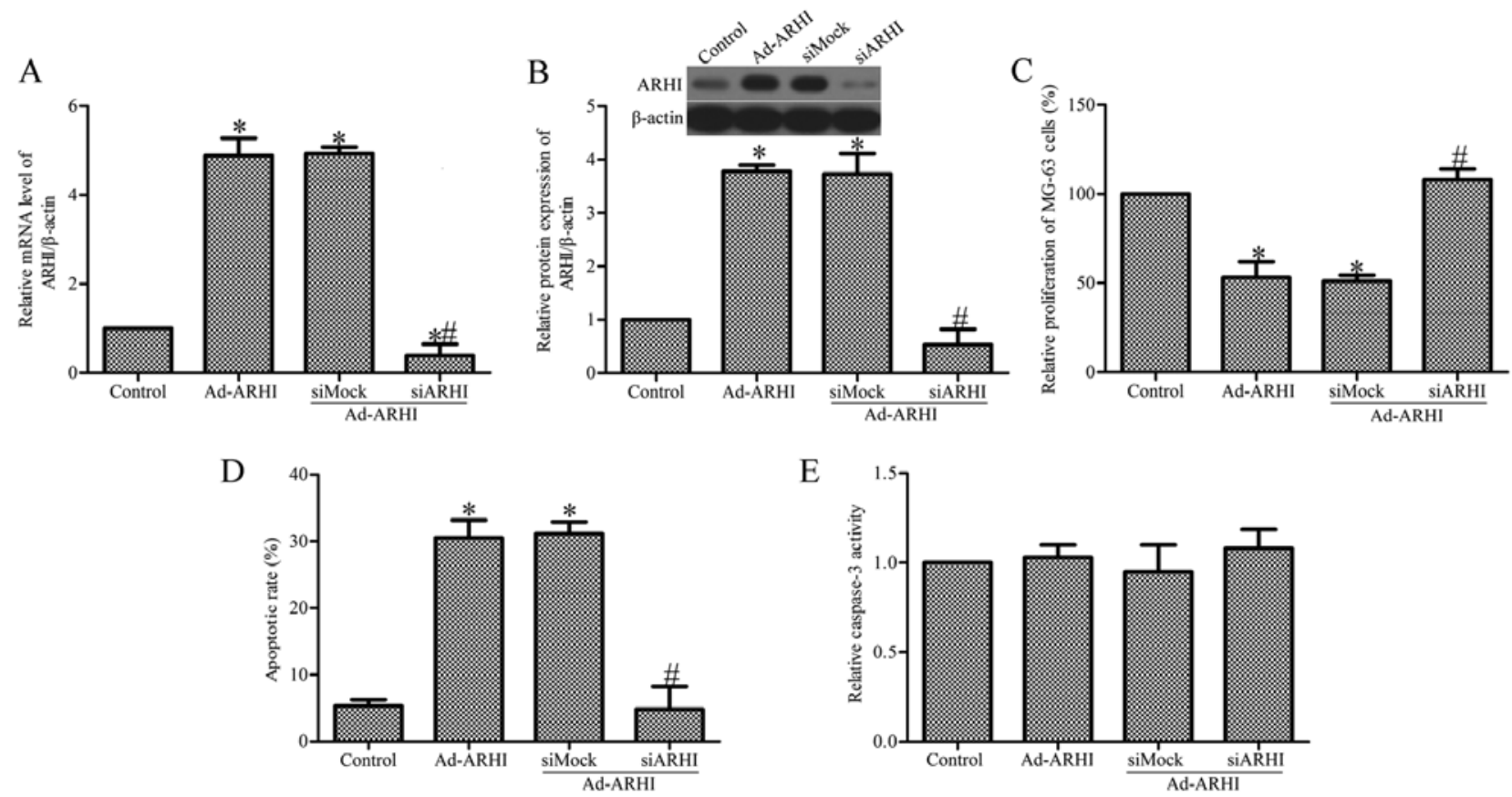

Figure 5. Effect of aplasia Ras homologue member I (ARHI) knockdown on tumor cell growth and apoptosis. (A) ARHI mRNA expression in MG-63 cells. (B) ARHI protein expression in MG-63 cells. (C) Analysis of ARHI silencing on cell proliferation of MG-63 cells. (D) Effect of ARHI knockdown on MG-63 cell apoptosis. (E) Caspase-3 activation was detected by the Caspase-Glo ${ }^{\circledR}$ 3/7 assay. The experiments were repeated at least three times. Values obtained from three individual experiments are expressed as mean \pm standard deviation, ${ }^{*} \mathrm{P}<0.05$ vs. control, ${ }^{\text {}} \mathrm{P}<0.05$ vs. Ad-ARHI group.

Specific inhibition of ARHI expression by ARHI-specific SiRNA. To further examine the role of ARHI in OS cell growth, Ad-AHRI-expressing MG-63 cells were transfected with siARHI or control siRNA (siMock). RT-qPCR and western blot analysis showed that the mRNA and protein expression of ARHI in the siARHI-transfected cells was markedly lower compared with the siMock and control groups (Fig. 5A and B). Transfection of siARHI evidently suppressed the proliferation of MG-63 cells (Fig. 5C). In addition, transfection of siARHI also significantly inhibited MG-63 cell apoptosis (Fig. 5D). To study the effect of siARHI on the activity of caspase-3 in Ad-AHRI-expressing MG-63 cells, the Caspase-Glo ${ }^{\circledR} 3 / 7$ assay was performed. The results indicate that transfection with siARHI did not affect caspase-3 activity. There was also no clear effect in the siMock group (Fig. 5E).

Overexpression of ARHI is directly associated with the inhibition of the cell survival pathway PI3K/Akt. Evidence shows that the PI3K/Akt signaling pathway is closely correlated with cancer cell biology, including OS $(34,35)$. In addition, Lu et al (36) showed that ARHI inhibits the PI3K/Akt signaling pathway in ovarian cancer cells. Thus, the effects of ARHI overexpression on the PI3K/Akt signaling cascade in MG-63 cells were examined. The results suggest that after Ad-ARHI was transfected into the OS cell line MG-63 for $72 \mathrm{~h}$, the expression level of p-Akt was markedly reduced in the Ad-ARHI group when compared with the levels in the Ad-Mock and control groups (Fig. 6). Notably, ablating the level of ARHI by siARHI markedly increased the activity of p-Akt compared with the Ad-ARHI group; however, the control siRNA showed no apparent difference (Fig. 6).

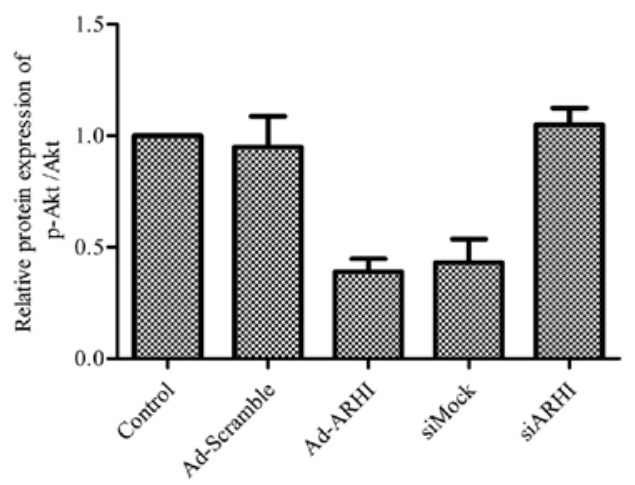

Figure 6. Effects of aplasia Ras homologue member I (ARHI) overexpression on the phosphorylation of Akt in MG-63 cells. The experiments were repeated at least three times. Values obtained from three individual experiments are expressed as mean \pm standard deviation, ${ }^{*} \mathrm{P}<0.05$ vs. control, ${ }^{\#} \mathrm{P}<0.05$ vs. Ad-ARHI group.

\section{Discussion}

Emerging studies have revealed that the identification of the specific target gene involved in tumorigenesis could provide valuable insight into the diagnosis and therapy of human malignancies (37). ARHI, a newly discovered, maternally imprinted tumor-suppressor gene, has been previously demonstrated to have an impact on growth, apoptosis, invasion, metastasis and tumor development in numerous types of cancers $(26,38)$. ARHI has been found to be reduced or absent in numerous tumors, while high expression of ARHI results in decreased cell proliferation, mainly through the induction of cell cycle-related genes $(23,26)$. The present study indicates 
that ARHI expression was reduced in the OS cell lines MG-63, U2OS and SAOS-2 compared to the human osteoblast precursor hFOB1.19 cell line (Fig. 1). ARHI overexpression was also shown to suppress cell viability and proliferation in these three OS cell lines (Fig. 2). In addition, ablating the expression of ARHI by siARHI significantly inhibited the growth of MG-63 cells (Fig. 4). Taken together, these results suggest that ARHI may act as a tumor-suppressor gene with an important role in the progression of OS. However, its roles in vivo require further study.

Increasing evidence has suggested that a high level of ARHI could lead to cancer cell apoptosis (14). It has been shown in other studies that significant overexpression of ARHI using an adenovirus vector induces caspase-independent apoptosis in ovarian and breast cancer cells (39). In the present study, Annexin V and PI staining indicated that the ARHI-expressing group had a significantly higher incidence of apoptosis compared with the Ad-Mock and control groups, and there was no apparent difference between the latter two groups (Fig. 3). In addition, knockdown of ARHI markedly suppressed MG-63 cell apoptosis in the ARHI-expressing group (Fig. 4). The effect of ARHI overexpression on the activity of caspase-3 was measured by the Caspase-Glo ${ }^{\circledR} 3 / 7$ assay. The results revealed that there was no clear difference between the Ad-ARHI, Ad-Mock and control groups; these results are consistent with those reported in the literature (39). These results indicate that ARHI-induced MG-63 cell apoptosis is independent of the caspase pathway; however, the specific mechanism of ARHI-induced apoptosis requires further study.

Numerous studies have established that ARHI induces tumor cell apoptosis, and excessive autophagy is closely associated with the PI3K/Akt signaling pathway $(12,36)$. In addition, the cell survival pathway PI3K/Akt has been implicated in tumor cell growth (40). In the present study, the findings indicate that overexpression of ARHI upregulated the expression of p-Akt in OS MG-63 cells, whereas knockdown of ARHI reduced the expression of $\mathrm{p}$-Akt in these cells, suggesting that ARHI may be indicated in the progression of OS via regulation of the PI3K/Akt pathway.

Taken together, there is a low level of ARHI in the OS cell lines. Overexpression of ARHI inhibited OS cell growth, induced cell apoptosis and suppressed PI3K/Akt signaling pathway activation. These results suggest that upregulation of ARHI expression is correlated with OS cell growth inhibition through inhibition of the PI3K/Akt pathway, and knockdown of ARHI promotes the growth of OS cells, suggesting that upregulation of ARHI may serve as a novel potential therapeutic target for the prevention and treatment of OS.

\section{Ackowledgements}

Financial support was provided by the Science and Technology Department of Gansu Province Natural Science Foundation Program (1208RJZA272).

\section{References}

1. Li HX, Meng QP, Liu W, Li YG, Zhang HM, Bao FC, Song LL and Li HJ: IMPDH2 mediate radioresistance and chemoresistance in osteosarcoma cells. Eur Rev Med Pharmacol Sci 18: 3038-3044, 2014.
2. Huh WW, Holsinger FC, Levy A, Palla FS and Anderson PM: Osteosarcoma of the jaw in children and young adults. Head Neck 34: 981-984, 2012.

3. Scholten DJ II, Timmer CM, Peacock JD, Pelle DW, Williams BO and Steensma MR: Down regulation of Wnt signaling mitigates hypoxia-induced chemoresistance in human osteosarcoma cells. PLoS One 9: e111431, 2014.

4. Mirabello L, Troisi RJ and Savage SA: International osteosarcoma incidence patterns in children and adolescents, middle ages and elderly persons. Int J Cancer 125: 229-234, 2009.

5. Ottaviani G and Jaffe N: The epidemiology of osteosarcoma. Cancer Treat Res 152: 3-13, 2009.

6. Shang HS, Chang JB, Lin JH, Lin JP, Hsu SC, Liu CM, Liu JY, Wu PP, Lu HF, Au MK, et al: Deguelin inhibits the migration and invasion of U-2 OS human osteosarcoma cells via the inhibition of matrix metalloproteinase-2/-9 in vitro. Molecules 19: 16588-16608, 2014.

7. Kuijjer ML, Hogendoorn PC and Cleton-Jansen AM: Genome-wide analyses on high-grade osteosarcoma: Making sense of a genomically most unstable tumor. Int J Cancer 133: 2512-2521, 2013.

8. Anninga JK, Gelderblom H, Fiocco M, Kroep JR, Taminiau AH, Hogendoorn PC and Egeler RM: Chemotherapeutic adjuvant treatment for osteosarcoma: Where do we stand? Eur J Cancer 47: 2431-2445, 2011.

9. Zuo X, Qin Y, Zhang X, Ning Q, Shao S, Luo M, Yuan N, Huang $S$ and Zhao X: Breast cancer cells are arrested at different phases of the cell cycle following the re-expression of ARHI. Oncol Rep 31: 2358-2364, 2014.

10. Li Y, Liu M, Zhang Y, Han C, You J, Yang J, Cao C and Jiao S: Effects of ARHI on breast cancer cell biological behavior regulated by microRNA-221. Tumour Biol 34: 3545-3554, 2013.

11. Fu Y, Chen J, Pang B, Li C, Zhao J and Shen K: EZH2-induced $\mathrm{H} 3 \mathrm{~K} 27 \mathrm{me} 3$ is associated with epigenetic repression of the ARHI tumor-suppressor gene in ovarian cancer. Cell Biochem Biophys 71: 105-112, 2015.

12. Li J, Cui G, Sun L, Wang SJ, Tian S, Guan Z, Fan WS, Yan ZF, Yang YZ, You YQ, et al: ARHI overexpression induces epithelial ovarian cancer cell apoptosis and excessive autophagy. Int $\mathrm{J}$ Gynecol Cancer 24: 437-443, 2014.

13. Huang J, Lin Y, Li L, Qing D, Teng XM, Zhang YL, Hu X, Hu Y, Yang P and Han ZG: ARHI, as a novel suppressor of cell growth and downregulated in human hepatocellular carcinoma, could contribute to hepatocarcinogenesis. Mol Carcinog 48: 130-140, 2009.

14. Wu X, Liang L, Dong L, Yu Z and Fu X: Effect of ARHI on lung cancer cell proliferation, apoptosis and invasion in vitro. Mol Biol Rep 40: 2671-2678, 2013.

15. Chen J, Shi S, Yang W and Chen C: Over-expression of ARHI decreases tumor growth, migration, and invasion in human glioma. Med Oncol 31: 846, 2014.

16. Ria R, Todoerti K, Berardi S, Coluccia AM, De Luisi A, Mattioli M, Ronchetti D, Morabito F, Guarini A, Petrucci MT, et al: Gene expression profiling of bone marrow endothelial cells in patients with multiple myeloma. Clin Cancer Res 15: 5369-5378, 2009.

17. Badgwell DB, Lu Z, Le K, Gao F, Yang M, Suh GK, Bao JJ, Das P, Andreeff M, Chen W, et al: The tumor-suppressor gene ARHI (DIRAS3) suppresses ovarian cancer cell migration through inhibition of the Stat 3 and FAK/Rho signaling pathways. Oncogene 31: 68-79, 2012.

18. Yu Y, Xu F, Peng H, Fang X, Zhao S, Li Y, Cuevas B, Kuo WL, Gray JW, Siciliano M, et al: NOEY2 (ARHI), an imprinted putative tumor suppressor gene in ovarian and breast carcinomas. Proc Natl Acad Sci USA 96: 214-219, 1999.

19. Prager GW, Poettler M, Unseld M and Zielinski CC: Angiogenesis in cancer: anti-VEGF escape mechanisms. Transl Lung Cancer Res 1: 14-25, 2011

20. Zou CF, Jia L, Jin H, Yao M, Zhao N, Huan J, Lu Z, Bast RC Jr, Feng Y and Yu Y: Re-expression of ARHI (DIRAS3) induces autophagy in breast cancer cells and enhances the inhibitory effect of paclitaxel. BMC Cancer 11: 22, 2011.

21. Méndez M, Custodio A and Provencio M: New molecular targeted therapies for advanced non-small-cell lung cancer. J Thorac Dis 3: 30-56, 2011.

22. Waltering KK, Helenius MA, Sahu B, Manni V, Linja MJ, Jänne OA and Visakorpi T: Increased expression of androgen receptor sensitizes prostate cancer cells to low levels of androgens. Cancer Res 69: 8141-8149, 2009. 
23. Lin D, Cui F, Bu Q and Yan C: The expression and clinical significance of GTP-binding RAS-like 3 (ARHI) and microRNA 221 and 222 in prostate cancer. J Int Med Res 39: 1870-1875, 2011.

24. Chen MY, Liao WS, Lu Z, Bornmann WG, Hennessey V, Washington MN, Rosner GL, Yu Y, Ahmed AA and Bast RC Jr: Decitabine and suberoylanilide hydroxamic acid (SAHA) inhibit growth of ovarian cancer cell lines and xenografts while inducing expression of imprinted tumor suppressor genes, apoptosis, G2/M arrest, and autophagy. Cancer 117: 4424-4438, 2011.

25. Lu X, Qian J, Yu Y, Yang H and Li J: Expression of the tumor suppressor ARHI inhibits the growth of pancreatic cancer cells by inducing G1 cell cycle arrest. Oncol Rep 22: 635-640, 2009.

26. Janssen EA, Øvestad IT, Skaland I, Søiland H, Gudlaugsson E, Kjellevold KH, Nysted A, Søreide JA and Baak JP: LOH at 1p31 (ARHI) and proliferation in lymph node-negative breast cancer. Cell Oncol 31: 335-343, 2009.

27. Zhao X, Li J, Zhuo J and Cai L: Reexpression of ARHI inhibits tumor growth and angiogenesis and impairs the mTOR/VEGF pathway in hepatocellular carcinoma. Biochem Biophys Res Commun 403: 417-421, 2010.

28. Visser S and Yang X: Identification of LATS transcriptional targets in HeLa cells using whole human genome oligonucleotide microarray. Gene 449: 22-29, 2010.

29. Li L, Luo J, Wang B, Wang D, Xie X, Yuan L, Guo J, Xi S, Gao J, Lin X, et al: Microrna-124 targets flotillin-1 to regulate proliferation and migration in breast cancer. Mol Cancer 12: 163, 2013

30. Li L, Yuan L, Luo J, Gao J, Guo J and Xie X: MiR-34a inhibits proliferation and migration of breast cancer through down-regulation of Bcl-2 and SIRT1. Clin Exp Med 13: 109-117, 2013.

31. Livak KJ and Schmittgen TD: Analysis of relative gene expression data using real-time quantitative PCR and the 2(-Delta Delta C(T)) method. Methods 25: 402-408, 2001.

32. Lu Z, Luo RZ, Lu Y, Zhang X, Yu Q, Khare S, Kondo S, Kondo Y, Yu Y, Mills GB, et al: The tumor suppressor gene ARHI regulates autophagy and tumor dormancy in human ovarian cancer cells. J Clin Invest 118: 3917-3929, 2008.

33. Jovicic A, Zaldivar Jolissaint JF, Moser R, Silva Santos MF and Luthi-Carter R: MicroRNA-22 (miR-22) overexpression is neuroprotective via general anti-apoptotic effects and may also target specific Huntington's disease-related mechanisms. PLoS One 8: e54222, 2013.
34. Zhou Y, Zhu LB, Peng AF, Wang TF, Long XH, Gao S, Zhou RP and Liu ZL: LY294002 inhibits the malignant phenotype of osteosarcoma cells by modulating the phosphatidylinositol 3-kinase/Akt/fatty acid synthase signaling pathway in vitro. Mol Med Rep 11: 1352-1357, 2015.

35. Zhang Y, Yang CQ, Gao Y, Wang C, Zhang CL and Zhou XH Knockdown of CXCR7 inhibits proliferation and invasion of osteosarcoma cells through inhibition of the PI3K/Akt and $\beta$-arrestin pathways. Oncol Rep 32: 965-972, 2014.

36. Lu Z, Yang H, Sutton MN, Yang M, Clarke CH, Liao WS and Bast RC Jr: ARHI (DIRAS3) induces autophagy in ovarian cancer cells by downregulating the epidermal growth factor receptor, inhibiting PI3K and Ras/MAP signaling and activating the FOXo3a-mediated induction of Rab7. Cell Death Differ 21: 1275-1289, 2014.

37. Gao Y, Luo LH, Li S and Yang C: miR-17 inhibitor suppressed osteosarcoma tumor growth and metastasis via increasing PTEN expression. Biochem Biophys Res Commun 444: 230-234, 2014

38. Papadimitriou K, Ardavanis A and Kountourakis P: Neoadjuvant therapy for locally advanced breast cancer: Focus on chemotherapy and biological targeted treatments' armamentarium. J Thorac Dis 2: 160-170, 2010.

39. Bao J-J, Le X-F, Wang R-Y, Yuan J, Wang L, Atkinson EN, LaPushin R, Andreeff M, Fang B, Yu Y, et al: Reexpression of the tumor suppressor gene ARHI induces apoptosis in ovarian and breast cancer cells through a caspase-independent calpain-dependent pathway. Cancer Res 62: 7264-7272, 2002

40. Yu P, Ye L, Wang H, Du G, Zhang J, Zhang J and Tian J: NSK-01105 inhibits proliferation and induces apoptosis of prostate cancer cells by blocking the Raf/MEK/ERK and PI3K/Akt/mTOR signal pathways. Tumour Biol 15: 15, 2014. 